\title{
RCS of Chiral Elliptic Cylinder Embedded in Infinite Chiral Medium
}

\author{
A-K. Hamid, W. Obaid \\ Department of Electrical and Computer Engineering, College of Engineering, University of Sharjah, Sharjah, UAE
}

\begin{tabular}{l}
\hline \hline Article Info \\
\hline Article history: \\
Received Jan 29, 2017 \\
Revised Jun 10, 2017 \\
Accepted Aug 11, 2017 \\
\hline Keywords: \\
Chiral/chiral, \\
Elliptic cylinder, \\
Mathieu functions, \\
Radar cross section, \\
Scattering
\end{tabular}

\begin{abstract}
This paper presents an analytic solution to the scattering properties of chiral elliptic cylinder embedded in infinite chiral medium due to incident plane wave. The external electromagnetic fields as well as the internal electromagnetic fields are written in terms Mathieu functions and expansion coefficients. In order to obtain both the internal and external unknown field expansion coefficients, the boundary conditions are applied rigorously at the surface of different chiral/chiral material. Results are plotted graphically for the normalized scattering widths for elliptic cylinders of different sizes and chiral materials to show the effects of these parameters on scattering cross widths. It is shown numerically by adding the external chiral material to elliptic cylinder provides more parameters to control the RCS.
\end{abstract}

Copyright () 2017 Institute of Advanced Engineering and Science. All rights reserved.

\section{Corresponding Author:}

\section{A-K. Hamid,}

Department of Electrical and Computer Engineering,

University of Sharjah,

Box 27272, Sharjah, UAE.

Email: Akhamidl@Sharjah.ac.ae

\section{INTRODUCTION}

It is well known that a reciprocal and isotropic chiral medium is characterized by different phase velocities for right- and left-circularly polarized (RCP and LCP) waves. In a lossless isotropic chiral medium, a linearly polarized wave undergoes a rotation of its polarization while it propagates. Numerous developments linked to chiral media overall are described in [1-11], and some analytical and numerical solutions to scattering from various types of chiral objects are given in [12-21].

The elliptic cylinder is a geometry that has been extensively analyzed in the literature due to its ability to create cylindrical cross sections of different shapes by changing the axial ratio of the ellipse. Furthermore, since the elliptic cylindrical coordinate system is one of the coordinate systems in which the wave equation is separable, solutions to problems involving elliptic cylinders can be obtained in exact form.

In this paper, we present a solution to the problem of scattering of a right-circularly polarized (RCP) plane wave from a chiral elliptic cylinder of arbitrary axial ratio placed in a distinct infinite chiral medium, while in [21] the chiral cylinder was embedded in free space. Both chiral media in this problem are isotropic. The present solution will require expanding the fields in two different chiral regions, and the solution obtained will therefore supply more parameters to control the normalized bistatic scattering width when compared to that in [21].

\section{THEORY}

Consider a RCP plane wave that is propagating in an infinite isotropic chiral medium, being incident on an infinitely long elliptic cylinder at an angle $\varphi_{i}$ with respect to the minus x-axis of a Cartesian coordinate system located at the center of a cross section of the cylinder with its z-axis along the axis of the 
cylinder, which is made up of a different chiral material and of major axis length $2 a$ and minor axis length $2 b$. For a RCP plane wave of amplitude $E_{0}$, the incident electric field can be written as

$$
\mathbf{E}^{i}=E_{0}\left(\hat{z}-j \hat{\varphi}_{i}\right) \exp \left[j k_{R 1} \rho \cos \left(\varphi-\varphi_{i}\right)\right]
$$

where $\hat{\kappa}$ is a unit vector in the direction of increasing $\kappa, k_{R 1}$ is the wavenumber of the incident plane wave, and $(\rho, \varphi)$ are the polar coordinates of an observation point with respect to the above mentioned Cartesian coordinate system. In accordance with the Drude-Born-Federov (DBF) constitutive relations that have been used in this paper [22], the wavenumber of the right circularly polarized wave is given by

$$
k_{R 1}=\frac{k_{0} \sqrt{\mu_{r 1} \varepsilon_{r 1}}}{1-k_{0} \gamma_{1} \sqrt{\mu_{r 1} \varepsilon_{r 1}}}
$$

where $k_{0}$ is the wavenumber in free space, $\gamma_{1}$ is the chirality parameter of the external chiral medium, and $\mu_{r 1}$ and $\varepsilon_{r 1}$ are the relative permeability and relative permittivity of the external chiral medium. The incident electric field in (1) can now be expanded in terms of elliptical vector wave functions as

$$
\mathbf{E}^{\mathrm{i}}=\sum_{m=0,1}^{\infty} A_{\substack{e m \\ o m}}\left[\mathbf{N}_{\substack{e m \\ o m}}^{(1)}\left(c_{R 1}, \mathbf{r}\right)+\mathbf{M}_{\substack{e m \\ o m}}^{(1)}\left(c_{R 1}, \mathbf{r}\right)\right]
$$

in which

$$
A_{\substack{e m \\
o m}}=E_{0} j^{m} \frac{\sqrt{8 \pi}}{N_{\substack{e m \\
o m}}\left(c_{R 1}\right)} S_{\begin{array}{c}
e m \\
o m
\end{array}}\left(c_{R 1}, \cos \varphi_{i}\right)
$$

where

$$
N_{\substack{e m \\ o m}}\left(c_{R 1}\right)=\int_{0}^{2 \pi}\left[S_{\substack{e m \\ o m}}\left(c_{R 1}, \cos v\right)\right]^{2} d v
$$

with $c_{R 1}=k_{R 1} F, S_{q n}\left(c_{\alpha}, \cos v\right)$ for $q=e, o$ being the angular Mathieu function of order $n$ and arguments $c_{\alpha}$ and $\cos v$, and $F$ being the semi-focal length of the cylinder. $\boldsymbol{\Xi}_{q m}^{(i)}\left(c_{\sigma}, \mathbf{r}\right)$ for $q=e, o$, and $\boldsymbol{\Xi}=\mathbf{M}, \mathbf{N}$ are defined in [23-24] in terms of angular and radial Mathieu functions, with $\mathbf{r}$ designating the elliptic coordinate dyad $(\xi, \eta)$. The summation over $m$ in (3) starts from 0 for even $(e)$ functions and from 1 for odd $(o)$ functions, and is the same for the other field expansions given below too. Maxwell's equations can next be used to expand the incident magnetic field in terms of elliptical vector wave functions as

$$
\mathbf{H}^{\mathrm{i}}=\frac{j}{Z_{1}} \sum_{m=0,1}^{\infty} A_{\substack{e m \\ o m}}\left[\mathbf{N}_{\substack{e m \\ o m}}^{(1)}\left(c_{R 1}, \mathbf{r}\right)+\mathbf{M}_{\substack{e m \\ o m}}^{(1)}\left(c_{R 1}, \mathbf{r}\right)\right]
$$

where $Z_{1}=Z_{0} \sqrt{\mu_{r 1} / \varepsilon_{r 1}}$, with $Z_{0}$ denoting the free space wave impedance. As the elliptic cylinder is made up of an isotropic chiral material and is surrounded by another isotropic chiral medium, the scattered and transmitted electro-magnetic fields will have both co-polar and cross-polar components. The scattered fields can hence be written as $\mathbf{E}^{\mathrm{s}}=\mathbf{E}_{\mathrm{cp}}^{\mathrm{s}}+\mathbf{E}_{\mathrm{xp}}^{\mathrm{s}}, \mathbf{H}^{\mathrm{s}}=j\left(\mathbf{E}_{\mathrm{cp}}^{\mathrm{s}}-\mathbf{E}_{\mathrm{xp}}^{\mathrm{s}}\right) / Z_{1}$, with co- and cross-polar fields $\mathbf{E}_{\mathrm{cp}}^{\mathrm{s}}, \mathbf{E}_{\mathrm{xp}}^{\mathrm{s}}$ expressed in terms of elliptical vector wave functions as

$$
\mathbf{E}_{\mathbf{c p}}^{\mathbf{s}}=\sum_{m=0,1}^{\infty} B_{\substack{e m \\ o m}}\left[\mathbf{N}_{\substack{e m \\ o m}}^{(4)}\left(c_{R 1}, \mathbf{r}\right)+\mathbf{M}_{e m}^{(4)}\left(c_{R 1}, \mathbf{r}\right)\right]
$$




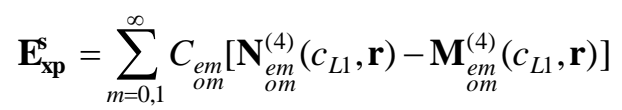

where $B_{q m}$ and $C_{q m}$ for $q=e, o$ are the unknown field expansion coefficients, $c_{L 1}=k_{L 1} F$, with the wavenumber $k_{L 1}$ for the left-circularly polarized wave given by

$$
k_{L 1}=\frac{k_{0} \sqrt{\mu_{r 1} \varepsilon_{r 1}}}{1+k_{0} \gamma_{1} \sqrt{\mu_{r 1} \varepsilon_{r 1}}}
$$

The fields transmitted inside the chiral elliptic cylinder can also be expressed as $\mathbf{E}^{\mathbf{c}}=\mathbf{E}_{\mathrm{cp}}^{\mathrm{c}}+\mathbf{E}_{\mathrm{xp}}^{\mathrm{c}}$, $\mathbf{H}^{\mathbf{c}}=j\left(\mathbf{E}_{\mathrm{cp}}^{\mathrm{c}}-\mathbf{E}_{\mathrm{xp}}^{\mathrm{c}}\right) / Z_{2}$, with $\mathbf{E}_{\mathrm{cp}}^{\mathrm{c}}, \mathbf{E}_{\mathrm{xp}}^{\mathrm{c}}$ expressed in terms of elliptical vector wave functions as

$$
\begin{aligned}
& \mathbf{E}_{\mathbf{c p}}^{\mathbf{c}}=\sum_{m=0,1}^{\infty} D_{\substack{e m \\
o m}}\left[\mathbf{N}_{\substack{e m \\
o m}}^{(1)}\left(c_{R 2}, \mathbf{r}\right)+\mathbf{M}_{\substack{e m \\
o m}}^{(1)}\left(c_{R 2}, \mathbf{r}\right)\right] \\
& \mathbf{E}_{\mathbf{x p}}^{\mathbf{c}}=\sum_{m=0,1}^{\infty} G_{\substack{e m \\
o m}}\left[\mathbf{N}_{\substack{e m \\
o m}}^{(1)}\left(c_{L 2}, \mathbf{r}\right)-\mathbf{M}_{\substack{e m \\
o m}}^{(1)}\left(c_{L 2}, \mathbf{r}\right)\right]
\end{aligned}
$$

where $D_{q m}$ and $G_{q m}$ for $q=e, o$ are the unknown field expansion coefficients, $c_{R 2}=k_{R 2} F$ and $c_{L 2}=k_{L 2} F$, with expressions for $k_{R 2}$ and $k_{L 2}$ obtained from (2) and (9), respectively, by changing the subscript 1 in these Equations, to 2. The unknown expansion coefficients can be obtained by imposing the boundary conditions corresponding to the continuity of the tangential field components at the surface $\xi=\xi_{\text {s }}$ of the chiral elliptic cylinder [25], which may be expressed mathematically as

$$
\begin{aligned}
& \left(\mathbf{E}^{\mathrm{i}}+\mathbf{E}^{\mathrm{s}}\right) \times\left.\hat{\xi}\right|_{\xi=\xi_{\mathrm{s}}}=\mathbf{E}^{\mathrm{c}} \times\left.\hat{\xi}\right|_{\xi=\xi_{\mathrm{s}}} \\
& \left(\mathbf{H}^{\mathrm{i}}+\mathbf{H}^{\mathrm{s}}\right) \times\left.\hat{\xi}\right|_{\xi=\xi_{\mathrm{s}}}=\mathbf{H}^{\mathrm{c}} \times\left.\hat{\xi}\right|_{\xi=\xi_{\mathrm{s}}}
\end{aligned}
$$

where $\hat{\xi}$ is the outward unit normal to the surface of the elliptic cylinder. Substituting the above developed expressions into the fields of (12) and (13), and applying the orthogonal property of the angular Mathieu functions, yield

$$
\begin{aligned}
& {\left[A_{q n} R_{q n}^{(1)}\left(c_{R 1}, \xi_{s}\right)+B_{q n} R_{q n}^{(4)}\left(c_{R 1}, \xi_{s}\right)\right] N_{q n}\left(c_{R 1}\right) } \\
+ & \sum_{m} C_{q m} R_{q m}^{(4)}\left(c_{L 1}, \xi_{s}\right) M_{q m n}\left(c_{L 1}, c_{R 1}\right) \\
= & \sum_{m}^{m} D_{q m} R_{q m}^{(1)}\left(c_{R 2}, \xi_{s}\right) M_{q m n}\left(c_{R 2}, c_{R 1}\right) \\
+ & \sum_{m} G_{q m} R_{q m}^{(1)}\left(c_{L 2}, \xi_{s}\right) M_{q m n}\left(c_{L 2}, c_{R 1}\right) \\
& {\left[A_{q n} R_{q n}^{(1)^{\prime}}\left(c_{R 1}, \xi_{s}\right)+B_{q n} R_{q n}^{(4)^{\prime}}\left(c_{R 1}, \xi_{s}\right)\right] N_{q n}\left(c_{R 1}\right) } \\
- & \frac{k_{R 1}}{k_{L 1}} \sum_{m} C_{q m} R_{q m}^{(4)^{\prime}}\left(c_{L 1}, \xi_{s}\right) M_{q m n}\left(c_{L 1}, c_{R 1}\right) \\
= & \frac{k_{R 1}}{k_{R 2}} \sum_{m} D_{q m} R_{q m}^{(1)^{\prime}}\left(c_{R 2}, \xi_{s}\right) M_{q m n}\left(c_{R 2}, c_{R 1}\right) \\
& -\frac{k_{R 1}}{k_{L 2}} \sum_{m} G_{q m} R_{q m}^{(1)^{\prime}}\left(c_{L 2}, \xi_{s}\right) M_{q m n}\left(c_{L 2}, c_{R 1}\right)
\end{aligned}
$$




$$
\begin{aligned}
& {\left[A_{q n} R_{q n}^{(1)}\left(c_{R 1}, \xi_{s}\right)+B_{q n} R_{q n}^{(4)}\left(c_{R 1}, \xi_{s}\right)\right] N_{q n}\left(c_{R 1}\right) } \\
& -\sum_{m} C_{q m} R_{q m}^{(4)}\left(c_{L 1}, \xi_{s}\right) M_{q m n}\left(c_{L 1}, c_{R 1}\right) \\
& =\frac{Z_{1}}{Z_{2}} \sum_{m} D_{q m} R_{q m}^{(1)}\left(c_{R 2}, \xi_{s}\right) M_{q m n}\left(c_{R 2}, c_{R 1}\right) \\
& -\frac{Z_{1}}{Z_{2}} \sum_{m} G_{q m} R_{q m}^{(1)}\left(c_{L 2}, \xi_{s}\right) M_{q m n}\left(c_{L 2}, c_{R 1}\right) \\
& {\left[A_{q n} R_{q n}^{(1)^{\prime}}\left(c_{R 1}, \xi_{s}\right)+B_{q n} R_{q n}^{(4)^{\prime}}\left(c_{R 1}, \xi_{s}\right)\right] N_{q n}\left(c_{R 1}\right) } \\
+ & \frac{k_{R 1}}{k_{L 1}} \sum_{m} C_{q m} R_{q m}^{(4)^{\prime}}\left(c_{L 1}, \xi_{s}\right) M_{q m n}\left(c_{L 1}, c_{R 1}\right) \\
= & \frac{k_{R 1} Z_{1}}{k_{R 2} Z_{2}} \sum_{m} D_{q m} R_{q m}^{(1)^{\prime}}\left(c_{R 2}, \xi_{s}\right) M_{q m n}\left(c_{R 2}, c_{R 1}\right) \\
+ & \frac{k_{R 1} Z_{1}}{k_{L 2} Z_{2}} \sum_{m} G_{q m} R_{q m}^{(1)^{\prime}}\left(c_{L 2}, \xi_{s}\right) M_{q m n}\left(c_{L 2}, c_{R 1}\right)
\end{aligned}
$$

for $q=e, o$ where $R_{q n}^{(i)}\left(c_{\alpha}, \xi_{s}\right)$ is the radial Mathieu function of order $n$ and kind $(i)$ of arguments $c_{\alpha}$ and $\xi_{s}$, and $M_{q m n}\left(c_{\alpha}, c_{\sigma}\right)$ is given by

$$
M_{q m n}\left(c_{\alpha}, c_{\sigma}\right)=\int_{0}^{2 \pi} S_{q m}\left(c_{\alpha}, \cos v\right) S_{q n}\left(c_{\sigma}, \cos v\right) d v
$$

The summations over $m$ in (14) - (17) runs from 0 to $N m a x-1$ for even $(e)$ functions, and from 1 to $N m a x$ for odd $(o$ ) functions, where Nmax is the number of terms needed to obtain a convergent solution. In (14)-(17), $n=0,1, \ldots, N \max -1$ or $n=1,2, \ldots, N \max$, according to whether $q=e$ or $q=o$. The system of equations in (14)-(17) can be expressed in matrix form and solved, to obtain the unknown field expansion coefficients. Using asymptotic expressions of the radial Mathieu functions of the fourth kind and their first derivatives, we can write the co- and cross polar scattered electric fields in the far zone as

$$
\begin{aligned}
& \mathbf{E}_{\mathrm{cp}}^{\mathrm{s}}=(\hat{z}+j \hat{\varphi}) \sqrt{\frac{j}{k_{R 1} \rho}} \exp \left(-j k_{R 1} \rho\right) \Omega_{B}(\varphi) \\
& \mathbf{E}_{\mathrm{xp}}^{\mathrm{s}}=(\hat{z}-j \hat{\varphi}) \sqrt{\frac{j}{k_{L 1} \rho}} \exp \left(-j k_{L 1} \rho\right) \Omega_{C}(\varphi)
\end{aligned}
$$

Where

$$
\Omega_{B}(\varphi)=\sum_{q=e, o} \sum_{m=0,1}^{\infty} j^{m} B_{q m} S_{q m}\left(c_{R 1}, \cos \varphi\right)
$$

$\Omega_{C}(\varphi)$ is obtained from (21), by changing $B$ to $C$ and $R 1$ to $L 1$. Explicit expressions for the normalized bistatic echo width of the right- and left-polarized waves can then be written as

$$
\frac{\sigma_{R}}{\lambda_{R}}=\left|\Omega_{B}(\varphi)\right|^{2}, \quad \frac{\sigma_{L}}{\lambda_{L}}=\left|\Omega_{C}(\varphi)\right|^{2}
$$

When $\varphi=\pi+\varphi_{i}$, (23) yields the exact normalized co- and cross-polar bistatic echo widths. 


\section{RESULTS AND ANALYSIS}

Numerical results are presented as normalized echo pattern widths for isotropic chiral elliptic cylinders of different axial ratios, embedded in another isotropic chiral medium of different relative permittivities and chirality parameters. First, we chose the parameters $\varepsilon_{r 1}=1.0, \mu_{r 1}=1.0, k_{0} \gamma_{1}=0.0$ for the exterior region, $k_{0} a=0.16 \pi \varepsilon_{r 2}=4.0, \mu_{r 2}=2.0, k_{0} \gamma_{2}=0.15$, and axial ratio $a / b=1.001$ for the cylinder, and $\varphi_{i}=180^{\circ}$. To validate the analysis and the software used for calculating the results, we computed the normalized echo pattern widths for the above chiral cylinder when it is excited by a plane wave that is transverse magnetically (TM) polarized in the axial z-direction, that is obtained by summing right- and leftcircularly polarized incident waves of equal field amplitudes. The results are in good agreement with those in [15] for an analogous chiral circular cylinder in free space, verifying the accuracy of the analysis and that of the software used for the calculations.

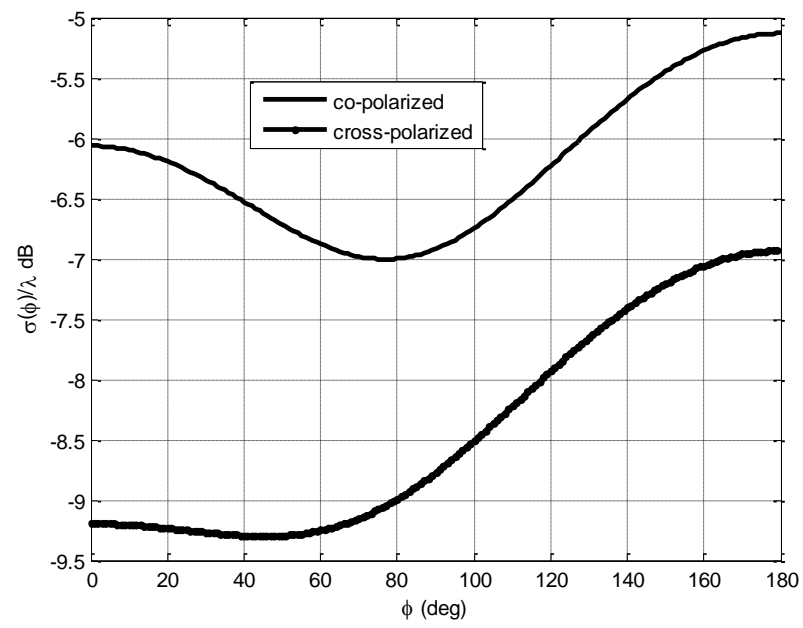

Figure 1. Normalized co-polar and cross polar bistatic scattering widths against the scattering angle, for a chiral elliptic cylinder of axial ratio $a / b=1.001$, with $k_{0} a=0.16 \pi \quad \varepsilon_{r 2}=4.0, \mu_{r 2}=2.0$, and $k_{0} \gamma_{2}=0.15$, and located in free space, when it is excited by a TM polarized plane wave incident at the angle $\varphi_{i}=180^{\circ}$.

Figure 2 displays the normalized right- and left-polarized echo-width patterns for a chiral elliptic cylinder of axial ratio 2 , with parameters $k_{0} a=0.16 \pi, \varepsilon_{r 2}=4.0, \mu_{22}=2.0$, and $k_{0} \gamma_{2}=0.15$, when it is embedded in a chiral medium having parameters $\varepsilon_{r 1}=1.0, \mu_{r 1}=1.0$, and $k_{0} \gamma_{1}=0.1$, and excited by a RCP plane wave incident at the angle $\varphi_{i}=180^{\circ}$. In this figure, the dominant right-polarized echo-width magnitude decreases gradually as the scattering angle increases from $0^{\circ}$ to $180^{\circ}$, but the corresponding left-polarized one increases first from $0^{\circ}$ to $120^{\circ}$, and then decreases.

Figure 3 shows the right- and left-polarized echo-width patterns for the chiral elliptic cylinder in Figure 2, when it is placed in a chiral medium which is similar to that in Figure 2, but with $\varepsilon_{r 1}=2.5$. When compared with the plots in Figure 2, we see that as the scattering angle increases from $0^{\circ}$ to $180^{\circ}$, the reduction of the right-polarized echo-width magnitude is much higher. Also the left-polarized echo-width magnitude is much lower for all scattering angles, though its behavior is somewhat similar to that of the same in Figure 2. 


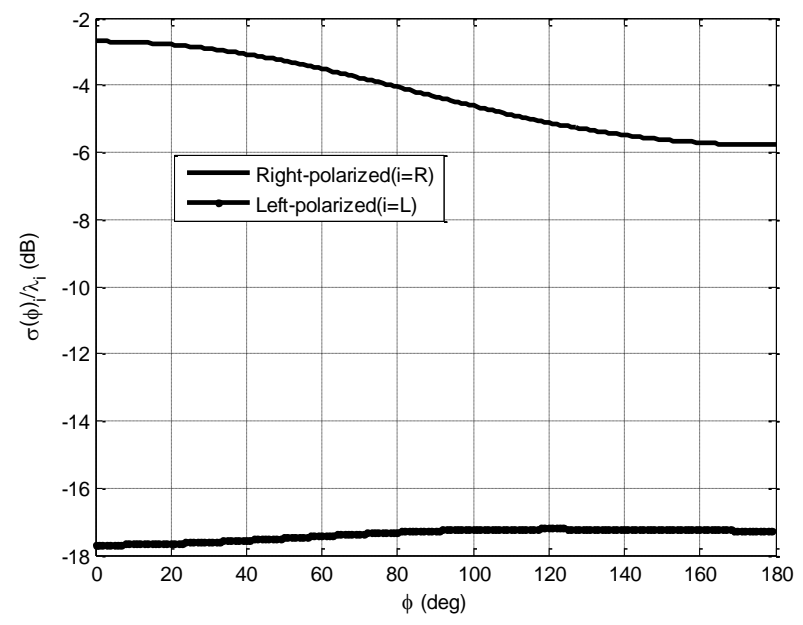

Figure 2. Normalized right- and left-polarized bistatic scattering widths against the scattering angle for a chiral elliptic cylinder of axial ratio 2.0, and having the same parameters as those for Figure 1, but located in a chiral medium with parameters $\varepsilon_{r 1}=1.0, \mu_{r 1}=1.0$, and $k_{0} \gamma_{1}=0.1$, when it is excited by a RCP plane wave incident at an incident angle of $180^{\circ}$.

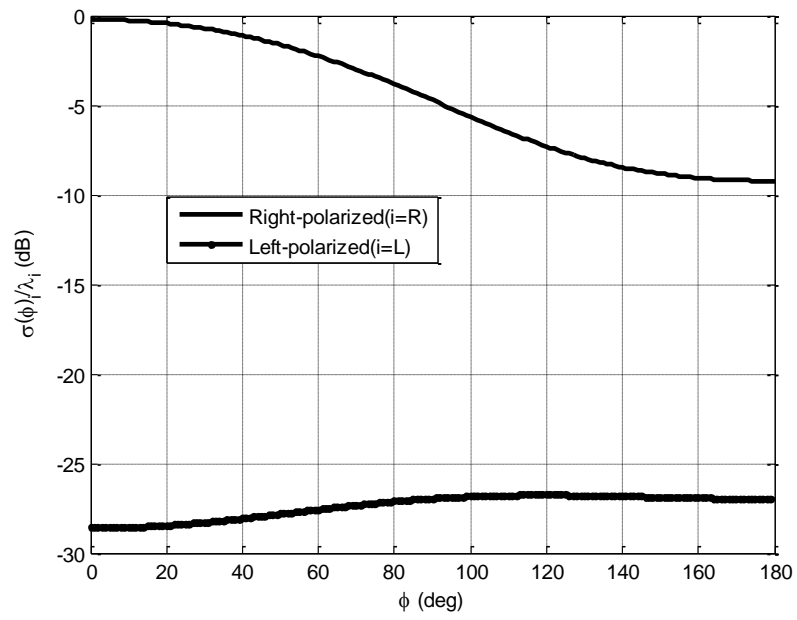

Figure 3. Normalized right- and left-polarized bistatic scattering widths against the scattering angle for the chiral elliptic cylinder in Figure 2, when it is placed in a chiral medium which is similar to that in Figure 2, but with $\varepsilon_{r 1}=2.5$, and illuminated by a RCP plane wave incident at $180^{\circ}$.

\section{CONCLUSION}

An exact solution to the problem of scattering of a RCP plane wave by a chiral elliptic cylinder placed in another chiral medium, presented using the method of separation of variables. Results have been presented as normalized bistatic right- and left-polarized echo-width patterns for chiral elliptic cylinders of different axial ratios and chiral materials, to show the effects of these on scattering. It is seen that the presence of two different chiral materials could significantly influence the left- and right-polarized pattern widths.

\section{ACKNOWLEDGEMENTS}

Prof. A-K. Hamid would like to acknowledge the support given by the University of Sharjah, Sharjah, United Arab Emirates. 


\section{REFERENCES}

[1] D. L. Jaggard, A. R. Mickelson, and C. H. Papas, "On electromagnetic waves in chiral media," Appl. Phys., vol. 18, pp. 211-216, 1979 .

[2] N. Engheta and P. Pelet, "Modes in chiro-waveguides," Opt. Lett., vol. 14, pp. 593-595, June 1989.

[3] C. Eftimiu and L. W. Pearson, "Guided electromagnetic waves in chiral media," Radio Sci., vol. 24, pp. 351-359, May-June 1989.

[4] N. Engheta and S. Bassiri, "One- and two-dimensional dyadic Green's functions in chiral media," IEEE Trans. Antennas Propagat., vol. 37, pp. 512-515, Apr. 1989.

[5] N. Engheta and D. L. Jaggard, "Electromagnetic chirality and its applications,', IEEE Antennas Propagat. Soc. Newsletter, vol. 30, pp. 6-12, Oct. 1988.

[6] S. Bassiri, C. H. Papas, and N. Engheta, "Electromagnetic wave propagation through a dielectric-chiral interface and through a chiral slab,”J. Opt. Soc. Am. A, vol. 5, pp. 1450-1459, Sept. 1988.

[7] W. S. Weiglhofer, "Isotropic chiral media and scalar Hertz potentials," J. Phys. A, vol. 21, pp. 2249-2251, 1988.

[8] D. L. Jaggard, X. Sun, and N. Engheta, "Canonical sources and duality in chiral media," IEEE Trans. Antennas Propagat., vol. 36, pp. 1007-1013, July 1988.

[9] A. Lakhtakia, V. V. Varadan, and V. K. Varadan, "Field equations, Huygens's principle, integral equations, and theorems for radiation and scattering of electromagnetic waves in isotropic chiral media," J. Opt. Soc. Am. A, vol. 5, pp. 175-184, Feb. 1988.

[10] A. Lakhtakia, V. V. Varadan, and V. K. Varadan, "Radiation by a straight thin-wire antenna embedded in an isotropic chiral media," IEEE Trans. Electromagn. Compat., vol. 30, pp. 84-87, Feb. 1988.

[11] B. N. Khatir and A. R. Sebak, "Slot antenna on a conducting elliptic cylinder coated by nonconfocal chiral media," Prog. Electromag. Res. (PIER), vol. 93, pp. 125-143, 2009.

[12] M. S. Kluskens and E. H. Newman, "Scattering by a multilayer chiral cylinder," IEEE Trans. Antennas Propagat., vol. 39, pp. 91 - 96, 1991

[13] A. Z. Elsherbeni, M. H. Al Sharkawy, and S. F. Mahmoud, "Electromagnetic scattering from a 2-D chiral strip simulated by circular cylinders for uniform and non-uniform chirality distribution," IEEE Trans. Antennas Propagat, vol. 52, pp. 2244-2252, 2004.

[14] S. Ahmed and Q. A. Naqvi, "Electromagnetic scattering from a chiral coated nihility cylinder," Progress in Electromag. Res. Lett., vol. 18, pp. 41-50, 2010.

[15] R. G. Rojas, "Integral equations for EM scattering by homo-geneous/inhomogeneous two-dimensional chiral bodies", IEE Proc. Microwave Antennas Propag., vol. 141, pp. 385-392, 1994.

[16] M. A. Al-Kanhal and E. Arvas, "Electromagnetic scattering from a chiral cylinder of arbitrary cross section," IEEE Trans. Antennas Propag., vol. 44, pp. 1041-1049, Jul. 1996.

[17] A. Semichaevsky, A. Akyurtlu, D. Kern, D. H. Werner, and M. G. Bray, Novel BI-FDTD approach for the analysis of chiral cylinders and spheres,” IEEE Trans. Antennas Propagat., vol. 54, pp. 925-932, 2006.

[18] S. Shoukat, S. Ahmed, M. Ashraf, A. Syed and Q. Naqvi, "Scattering of electromagnetic plane wave from a chiral cylinder placed in a chiral metamaterial," J. Electromag. Waves Applic., vol. 27, pp. 1127-1135, 2013.

[19] B. N. Khatir, M. Al-Kanhal, and A. Sebak, "Electromagnetic wave scattering by elliptic chiral cylinder," J. Electromag. Waves Applic., vol. 20, pp. 1377-1390, 2006.

[20] A-K. Hamid, "EM scattering by a lossy dielectric-coated nihility elliptic cylinder," Appl. Comp. Electromag. Soc. (ACES) Journal, vol. 25, pp. 444-449, 2010.

[21] A-K. Hamid, "Scattering by chiral lossy metamaterial elliptic cylinders" Appl. Comp. Electromag. Soc. Journal, vol. 27, pp. 603-609, 2012.

[22] I. V. Lindell, A. H. Sihvola, S. A. Tretyakov, and A. J. Viitanen, Electromagnetic Waves in Chiral and Bi-isotropic Media, Artech House, Boston, USA, 1994.

[23] A.-K. Hamid and F. R. Cooray, "Scattering from a chirally coated DB elliptic cylinder," AËU, vol. 68, pp. 1106$1111,2014$.

[24] A.-K. Hamid and F. R. Cooray, "Two-dimensional scattering by a homogeneous gyrotropic-type elliptic cylinder," Advan. Electromag., vol. 5, pp. 106-112, Dec. 2016.

[25] A.-K. Hamid, "Scattering by a chiral elliptic cylinder placed in another infinite chiral medium," Appl. Comp. Electromag. Soc. Conf., Florence, March, 2017 